Results The prevalence of depression and depressive symptoms were $4.8 \%$ and $21.4 \%$, respectively. Depression (OR 8.0; 95\% CI 4.2 to 14.3 ) and depressive symptoms (OR 2.7; $95 \%$ CI 1.7 to 4.2 ) were strongly associated with high disability, even after adjustment for all demographic and socioeconomic conditions and physical morbidities. Depression had a PAF of $15 \%$ (95\% CI $10 \%$ to $19 \%)$, whereas depressive symptoms had a PAF of $17 \%$ (95\% CI $9 \%$ to $24 \%$ ).

Conclusions Depression and depressive symptoms were the morbidities that contributed most to the framework of disability in the elderly. Effective management of depressive states in the elderly, delivered at the primary care level, may reduce the total population disability.

\section{P2-510 GESTATIONAL AND NEONATAL OUTCOMES IN TEENAGE PREGNANCIES}

doi:10.1136/jech.2011.142976m.37

${ }^{1} \mathrm{~A}$ Dias, ${ }^{* 1,2}$ E L Costa, ${ }^{2} \mathrm{M}$ C F Sena, ${ }^{1} \mathrm{~L}$ S Vianna. ${ }^{1}$ Botucatu Medical School, Botucatu, Sao Paulo, Brazil; ${ }^{2}$ Health Sciences School, Brasilia, Federal District, Brazil

Introduction According to the UK government, social exclusion increases the risk of teenage pregnancy and educational factors may be dimensions of such exclusion. In Brazil, around 700000 girls (32000 under 14 years) were reported to give birth in 1999.

Objective To compare gestational follow-up and neonatal outcomes in the age groups of 12-14 years (early adolescence), 15-18 years (late adolescence), and 19-35 years (adulthood), based on the number of prenatal visits, birthweight, and 5-min Apgar score.

Method Retrospective cross-sectional study conducted between 1 January 2006 and 31 December 2009, using secondary data (Livebirth statement) from the South Wing Regional Hospital, Brasília, Federal District.

Results Of 24800 newborns, the mothers of $87.06 \%$ were adults, $12.25 \%$ late adolescents and $0.69 \%$ early adolescents. The number of prenatal visits was adequate ( $\geq 7$ visits) in $85.60 \%$ of the adults, and in only $14.00 \%$ and $0.40 \%$ of the late and early adolescents, respectively, with statistically significant differences. Birthweight distribution also significantly differed among groups. In adult pregnancies, birthweight values were the highest, although mean values were within the normal range. The proportion of low birthweight was significantly higher in early adolescence pregnancies. Five-minute Apgar scores were inadequate in $4.80 \%$ of early adolescence pregnancies, $2.80 \%$ of late adolescence pregnancies, and $1.90 \%$ of adult pregnancies, with distribution also differing among groups.

Conclusion Adolescent mothers require special care during gestation as they comprise a risk group that has been neglected. The adverse perinatal outcomes observed underscore the inadequacy of gestational follow-up.

\section{P2-511 THE RELATIONSHIP BETWEEN ALCOHOL USE AND INJECTING DRUG USE: IMPACTS ON HEALTH AND SOCIAL FUNCTIONING}

doi:10.1136/jech.2011.142976m.38

${ }^{1,2} \mathrm{P}$ Dietze, ${ }^{*}{ }^{1} \mathrm{R}$ Jenkinson, ${ }^{1} \mathrm{C}$ Aitken, ${ }^{1} \mathrm{M}$ Stoove, ${ }^{2} \mathrm{D}$ Jolley. ${ }^{1}$ Burnet Institute, Melbourne, Victoria, Australia; ${ }^{2}$ Monash University, Melbourne, Victoria, Australia

Introduction Alcohol is a major risk factor for heroin overdose, but the effects of alcohol consumption on other health and social outcomes for people who inject drugs have not been systematically evaluated. In this paper we explore the effects of alcohol on health and social outcomes for people who inject drugs.

Methods Data were obtained from 655 people who inject drugs as part of the Melbourne Injecting Drug User cohort study (MIX). AUDIT-C scores $(0,1-8,8+)$ were generated and associations between these AUDIT-C scores and health and social outcomes were examined using logistic regression for dichotomous outcomes and linear regression for continuous outcomes.

Results While around 40\% of the MIX cohort reported never drinking alcohol, 43\% scored between 1 and 8 and $17 \%$ above 8 on the AUDIT-C. A score of $8+$ on the AUDIT $C$ was associated with a variety of negative health outcomes including non-fatal heroin overdose, as well as increased use of health services such as hospital emergency departments. Participants who reported drinking were more likely to report perpetrating violent crime, but were no more likely to report committing other sorts of crime such as fraud. Drinkers were more likely to report less overall life satisfaction than non-drinkers

Conclusion The rate of abstinence in the MIX cohort was high, but those who reported drinking alcohol exhibited poorer health and social outcomes. Alcohol was associated with an increase in violent offending within a population with high rates of offending more generally.

\section{P2-512 PSYCHIATRIC READMISSIONS AND THEIR ASSOCIATION WITH SOCIODEMOGRAPHIC AND CLINICAL PROFILES}

doi:10.1136/jech.2011.142976m.39

${ }^{1}$ A R F Furegato, ${ }^{*}$ 'S A Castro, ${ }^{2} \mathrm{~J}$ L F Santos. ${ }^{1}$ Escola de Enfermagem de Ribeirão Preto - USP, Ribeirão Preto, São Paulo, Brazil; ${ }^{2}$ Faculdade de Medicina de Ribeirão Preto - USP, Ribeirão Preto, São Paulo, Brazil

Introduction Despite recent advances, the frequency of psychiatric hospitalisations remains high.

Objective To identify the frequency of readmissions in a psychiatric hospital associated with patients' social demographic and clinical profiles.

Methods A structured questionnaire was applied during 2008 to collect the reports from the Psychiatric Hospital at Ribeirão Preto - Brasil

Results In 2025 records, 681 readmissions (34\%) were identified. Most of the subjects ( $99 \%$ ) came from the same region of the study, the majority were men, white, $30 \%$ aged $40-49$ and $26 \%$ up to 29 years, $23 \%$ were married. Most of the readmissions were caused only by treatment dropout (47\%) or associated with low family support (26\%). Prevalent diagnoses were schizophrenia $(27 \%)$, affective disorders (30\%) and use of alcohol and drugs (15\%). The mean age at beginning of disease was higher among men $(p=0.001)$. The length of stay in the hospital was higher for women, especially in the age group 40 to $49(p=0.032)$. Associations were found between gender and age $(p=0.007)$, marital status $(p=0.000)$, reason for hospitalisation ( $p=0.002)$, physical status on admission $(p=0.004)$, hospitalisations in other psychiatric hospitals $(p=0.003)$, type of discharge $(p=0.003)$, treatment during hospitalisation and physical status at discharge $(\mathrm{p}=0.004)$.

Conclusions The flow of care is consistent with the principles of regionalisation of the Unified Health System (SUS), however, the rate of readmissions due to treatment dropout is very high, which is an epidemiological alert to all sectors involved.

\section{P2-513 SOCIAL AND GEOGRAPHICAL VARIATIONS OF MATERNAL MORTALITY IN MEXICO}

doi:10.1136/jech.2011.142976m.40

G J Gonzalez-Perez,* M G Vega-Lopez, C E Cabrera-Pivaral. University of Guadalajara, Guadalajara, Mexico

Introduction This paper analyses the trend of maternal mortality rate in Mexico according to the level of social marginalisation 
between 1990 and 2008 and identifies socioeconomic factors that better explain the geographical variations of this indicator around 2008.

Methods Maternal mortality rates were calculated by level of municipal social marginalisation during the studied period. In addition, maternal mortality rates by state were calculated in 2008; by a multiple linear regression analysis, the variables that better explain interstate variations in the rate were identified.

Results The results show that in the last 20 years the maternal mortality rate has barely declined in the country (current rate is around 60 by 100000 live births), and that the ratio between the extreme strata rates-very high vs very low marginalisation -has been increased from 2.7 to 3.4 in the last 10 years. In turn, are social factors linked to poverty and social exclusion (percentage of housings with dirt floor) as well as issues related to health services (per capita public expenditure in health or percentage of population without adequate healthcare) those who better explain the variations in maternal mortality among the states. The found model explains $70 \%$ of the interstate variations of the rate.

Conclusion Previously enunciated aspects reveal the role of social conditions in maternal health and especially, in the possibility of avoiding a death from complications of pregnancy, childbirth and postpartum and demonstrate the need to change the social environment of women to reduce maternal mortality rate in Mexico.

\section{P2-514 PHYSICAL CHILD MALTREATMENT AMONG ASYLUM SEEKERS IN THE NETHERLANDS; A NATIONWIDE MEDICAL RECORDS STUDY}

doi:10.1136/jech.2011.142976m.41

\begin{abstract}
${ }^{1,2}$ S Goosen, ${ }^{*}$ A Kunst. ${ }^{1}$ Netherlands Association for Community Health Services, Utrecht, The Netherlands; ${ }^{2}$ Department of Public Health, Academic Medical Centre, University of Amsterdam, Amsterdam, The Netherlands
\end{abstract}

Introduction Refugee children in Western countries are considered to be at increased risk for child maltreatment. Data about asylum seeker children, however, are rare. The aim of our study was to assess the registered incidence of physical child maltreatment among asylum seekers in the Netherlands between 2000 and 2008, and associations with demographic and reception factors.

Methods We used the electronic database of the community health services for asylum seekers, containing demographic, reception and health data of all asylum seekers in the Netherlands between 1 January 2000 and 31 December 2008. The study includes children $<18$ years in reception with at least one parent $(N=22456)$. We used Cox regression analysis.

Results The number of physical child maltreatment cases was 182. The registered incidence was 3.7 cases per 1000 person years. Multivariate Cox regression showed an increased risk for girls $(\mathrm{RR}=1.34 ; 95 \% \mathrm{CI} 0.99$ to 1.80$)$ and children in single parent units ( $R R=1.72,95 \%$ CI 1.25 to 2.37 ). Children born in reception have a lower incidence compared to children born before arrival. Those $12-18$ years at arrival had the largest risk $(R R=2.69,95 \%$ CI 1.57 to 4.59). Incidence differences were also found between countries of origin. The highest incidence was found for children from Iran and Former Yugoslavia $(\mathrm{RR}=1.49$; $95 \% \mathrm{CI} 0.84$ to 2.65 and $\mathrm{RR}=1.30$; $95 \%$ CI 0.72 to 2.34 ). Length of stay was not an independent risk factor.

Conclusion Our study shows differences in the risk for physical child maltreatment between asylum seeker subgroups. Girls, children in single parent units and those with higher age at arrival are at increased risk. The results will contribute to priority setting in child maltreatment prevention.

\section{P2-515 INVESTIGATING VICTORIA'S INVERSE EQUITY HYPOTHESIS: THE CHANGING SOCIAL EPIDEMIOLOGY OF HIV INFECTION IN TANZANIA}

doi:10.1136/jech.2011.142976m.42

1J Hargreaves, * ${ }^{2} \mathrm{~L}$ Howe, ${ }^{1} \mathrm{E}$ Slaymaker. 'London School of Hygiene and Tropical Medicine, London, UK; ${ }^{2}$ Department of Social Medicine, University of Bristol, Bristol, UK

Introduction Cesar Victora's "inverse equity hypothesis" predicts that socioeconomic inequality in a health outcome will increase as new interventions are introduced, but suggests the gap will close over time. We explored this in relation to the HIV epidemic in Tanzania.

Methods Literature review and analysis of two nationally-representative HIV prevalence surveys conducted among adults aged 15-49 in 2003-2004 ( $N=10934)$ and 2007-2008 ( $N=15542)$. We explored whether changes over time in HIV prevalence and sexual-behaviours differed according to socioeconomic position (measured by educational attainment). In our analysis we pooled the survey data and fitted time-period*SEP interactions within appropriately adjusted regression models for each outcome.

Results Literature review suggested that when HIV prevention activities were relatively undeveloped during the 1990s, HIV prevalence was higher among more educated individuals in Tanzania. However, between 2003/4 and 2007/8 HIV prevalence was stable among those with no education (adjusted OR 2007-2008 vs 2003-2004 1.03, 0.72-1.47), whereas prevalence declined among those with secondary education (adjusted OR $0.53,0.34-0.84)$. Time-trends by education differed with respect to different aspects of sexual behaviour. There was some evidence that the association between educational attainment and recent condom use was now moving from a large poor-rich gap to a smaller one.

Conclusion The data are partially supportive of the inverse equity hypothesis. The data suggest a widening over time of the poor-rich gap in prevalent HIV infections (which may have been acquired some time ago), but there was some evidence that trends in recent condom use reflect the final "gap closing" component.

\section{P2-516 EDUCATIONAL LEVEL AND OCCUPATIONAL SOCIAL CLASS AND THE INCIDENCE OF LIFE-STYLE RELATED CHRONIC DISEASES IN THE EUROPEAN PROSPECTIVE INVESTIGATION INTO CANCER AND NUTRITION (EPIC)- POTSDAM STUDY}

doi:10.1136/jech.2011.142976m.43

${ }^{1,2} \mathrm{~A}-\mathrm{K} \mathrm{IIIner},{ }^{*}{ }^{3} \mathrm{U}$ Noethlings, ${ }^{2} \mathrm{M}$ Bergmann, ${ }^{1} \mathrm{H}$ Boeing. ${ }^{1}$ Dietary Exposure Assessment Group, International Agency for Research on Cancer, Lyon, France; ${ }^{2}$ Department of Epidemiology, German Institute of Human Nutrition, Nuthetal, Germany; ${ }^{3}$ Section for Epidemiology, Institute for Experimental Medicine, University of Kiel, Kiel, Germany

Introduction Healthy lifestyle choices, such as weight control through a balanced diet and regular physical activity, play a key role in chronic disease prevention. Underlying socioeconomic factors may mediate disease risk, but are often neglected. We aimed to investigate whether either educational level or occupational social class influences the incidence of diabetes, myocardial infarction, stroke and cancer, independently from a healthy lifestyle.

Methods We prospectively examined the associations of both indicators with incident chronic diseases in 23453 participants, aged 35-65 of the EPIC-Potsdam Study. Age- and multivariate adjusted HRs (HR, 95\% CI) were calculated. An additional systematic literature search aimed to identify how different socioeconomic indicators were related to the selected diseases. 